\title{
Robustness of Decoherence-Free Subspaces for Quantum Computation
}

\author{
D. Bacon ${ }^{(1,2)}$, D.A. Lidar ${ }^{(2)}$, and K.B. Whaley ${ }^{(2)}$ \\ Department of Physics ${ }^{(1)}$ and Department of Chemistry ${ }^{(2)}$, \\ The University of California, Berkeley, CA 94720
}

(October 13, 2018)

\begin{abstract}
It was shown recently [D.A. Lidar et al., Phys. Rev. Lett. 81, 2594 (1998)] that within the framework of the semigroup Markovian master equation, decoherence-free (DF) subspaces exist which are stable to first order in time to a perturbation. Here this result is extended to the nonMarkovian regime and generalized. In particular, it is shown that within both the semigroup and the non-Markovian operator sum representation, DF subspaces are stable to all orders in time to a symmetry-breaking perturbation. DF subspaces are thus ideal for quantum memory applications. For quantum computation, however, the stability result does not extend beyond the first order. Thus, to perform robust quantum computation in DF subspaces, they must be supplemented with quantum error correcting codes.
\end{abstract}

\section{INTRODUCTION}

The power promised by quantum computers [1] has initiated an intense scrutiny of the physical viability of these computers [2 - The central obstacle in the experimental realization of such computers has proven to be maintaining the quantum coherence of states which form the cornerstone of the speedup promised by quantum computers. The main cause of this degradation of the quantum coherence is the coupling of the computer to the environment, and the subsequent decoherence induced by this coupling. To overcome this difficulty, Quantum Error Correcting Codes (QECC) inspired by classical coding theory have been developed [5]. These codes are "active" in the sense that decoherence is fought by continuous application of error correction procedures to quantum bits (qubits), which are encoded over the Hilbert space of several physical qubits. Another approach has emerged more recently, in which the structure of the physical decoherence process is used to protect the precious quantum coherence [6 11]. These "passive" error prevention codes rely on symmetries of the decoherence process to encode qubits into states which reside in the decoherence-free (DF) subspaces of multiple physical qubit systems. The conditions under which such DF subspaces can exist have been established in both the Lindblad (Markovian) formulation [9, 10] and for the nonMarkovian case [7].

The non-Markovian formulation of the reduced dynamics of a subsystem is used extensively in the quantum computation literature, where it is known as the "operator sum representation" (OSR) 12]. While it is exact, it is not always clear how to separate system from bath in this approach, since for any finite-dimensional bath the dynamics are reversible (albeit potentially with a very large Poincaré recurrence time) 13 as long as no measurements are made. In the Lindblad formulation, on the other hand, the dynamics being described are a priori that of the system alone, subject to preservation of complete positivity of the system density matrix. The dynamics are irreversible, but the price paid is that the Markovian approximation must be invoked [14]. The first aim of this paper is to clarify the relation between these two formulations. We do this with a derivation of the Lindblad semigroup master equation (SME) from the OSR in a way which highlights the differences and similarities between the two. Derivations of master equations are numerous, starting with the work of Zwanzig and others in the late 1950s |15]: our current derivation is novel in that it starts from a fixed-basis form of the OSR which is constructed to formally resemble the SME, yet is still exact. This fixed-basis representation allows one to clearly identify the manner in which the temporal coarse-graining assumption is invoked in the process of making the transition from non-Markovian to Markovian dynamics, as well as the consequences of this. The fixed-basis OSR equation leads to several important results concerning the decay of quantum coherence. First, we show that any finite total Hamiltonian will have a zero first order decoherence rate in the non-Markovian case, but that this feature can be destroyed by the coarse-grained time averaging made upon going to the Markovian limit. Second, a non-vanishing first order decay rate within the non-Markovian OSR formulation necessarily implies a singularity of the total Hamiltonian. We show explicitly how this accounts for the behavior seen in the prototypical example of phase damping, which is commonly used in the quantum computation literature.

The second, and main, focus of this paper is the use of the fixed-basis OSR representation to provide a general stability analysis of DF subspaces. In Ref. 10] it was shown that, within the SME, DF subspaces are stable to symmetry-breaking perturbations, to first order in time. This leads to a lowering of the threshold for fault-tolerant quantum computation [16,17] in DF subspaces. This stability result was obtained for the "memory-fidelity", i.e., 
for a quantum computer not subject to external "programming pulses". Since DF subspaces also appear in the non-Markovian setting [7,11], it is natural to enquire then whether the robustness of DF subspaces with respect to perturbations extends also to the non-Markovian situation. We make a stability analysis within the OSR here to address this, and find that the robustness of the DF subspaces is upheld in the more general situation. Indeed, we show that in fact, within both the SME and the OSR, DF subspaces are stable to a symmetry-breaking perturbation to all orders of time.

The generality of this stability result for memory fidelity does not extend however to the "dynamical fidelity", which measures the preservation of quantum coherence in the case of a quantum computer that is subject to external programming pulses. We examine this dynamical fidelity here and find that in this case, a DF subspace is stable only to first order in time. Thus while a DF subspace can drastically extend the decoherence time for quantum memory, operations performed on the DF subspace must be performed rapidly (in comparison to the perturbing error rate) in order achieve a similar extension of the decoherence time for implementing quantum computation. Barring methods that rely on symmetrizing operations on a time-scale faster than the primary decoherence rate [18], DF subspaces were, however, never meant to be a complete solution to the problem of decoherence on a quantum computer. Their usefulness lies in the elimination of the primary source of decoherence and the subsequent lengthening of the decoherence time to one determined solely by the decoherence due to perturbing errors. DF subspaces should be supplemented by a QECC in order to achieve a decoherence free quantum computer. This is possible using a concatenation of DF and QECC codes, as was shown in Ref. [11]. The instability of DF subspaces while the system is evolving thus sets a lower bound on how rapidly operations on the DF subspace must be performed in order realize the robustness of DF subspaces.

The structure of the paper is as follows. In Sec. IIA a brief review of the OSR formalism is presented, followed by the derivation of the fixed basis form for the OSR equation in Sec. IIB. This equation is used to derive the SME in Sec. II C. In Sec. III we turn to the main subject of the paper, namely short-time expansions of the fidelity. Addressing first the fidelity over the entire system Hilbert space, we derive the first order decoherence rate within the OSR and show that it vanishes generally in the OSR for non-Markovian dynamics, provided that the total Hamiltonian is of a non-singular form. This condition precludes the situation of a system coupled to an infinite number of degrees of freedom. We then show that this first order rate may become finite as a result of the coarse-grained time averaging performed on going to the Markovian limit. The generality of the non-Markovian result appears initially surprising, since there exist elementary examples of non-vanishing first order decoherence rates in the non-Markovian situation. In Sec. IIIB, we show with the example of the well-known case of decoherence due to phase damping, how this reflects an underlying singularity in the total Hamiltonian. Sec. [V] then deals with the special issue of stability for the DF subspaces. After a brief summary of the conditions for DF subspaces in the two approaches, we then show that DF subspaces have enhanced stability over the general system Hilbert space, namely that they are stable to all orders of symmetry-breaking perturbations, both within the non-Markovian OSR and the Markovian (SME) limit. In Sec. V, we then address the "dynamical fidelity" of DF subspaces under external fields, corresponding to a "quantum computer program." We show that this is stable to a lesser extent, possessing a vanishing first order decoherence rate in both the SME and the OSR, but having non-zero terms of higher order in time. We conclude with a summary and discussion of the implications for quantum computation in Section VI.

\section{THE OPERATOR SUM REPRESENTATION}

\section{A. Brief Review}

The dynamics of a quantum system $S$ coupled to a bath $B$, which together form a closed system, evolves unitarily under the combined system-bath Hamiltonian $\mathbf{H}_{S B}=\mathbf{H} \otimes \mathbf{I}_{B}+\mathbf{I}_{S} \otimes \mathbf{H}_{B}+\mathbf{H}_{I}$. Here $\mathbf{H}, \mathbf{H}_{B}$ and $\mathbf{H}_{I}$ are, respectively, the system, bath and interaction Hamiltonians, and $\mathbf{I}$ is the identity operator. Assuming that $S$ and $B$ are initially decoupled, so that the total initial density matrix is a tensor product of the system and bath density matrices ( $\rho$ and $\rho_{B}$ respectively), the system dynamics are described by the reduced density matrix:

$$
\rho(0) \longmapsto \rho(t)=\operatorname{Tr}_{B}\left[\mathbf{U}\left(\rho \otimes \rho_{B}\right) \mathbf{U}^{\dagger}\right] .
$$

Here $\operatorname{Tr}_{B}$ is the partial trace over the bath and $\mathbf{U}=\exp \left(-\frac{i}{\hbar} \mathbf{H}_{S B} t\right)$. By using a spectral decomposition for the bath, $\rho_{B}=\sum_{\nu} \nu|\nu\rangle\langle\nu|$, and introducing a basis $\{|n\rangle\}_{n=1}^{N}$ for the $N$-dimensional system Hilbert space $\mathcal{H}$, this can be rewritten in the OSR as [12, 19]:

$$
\rho(t)=\sum_{i=0}^{K} \mathbf{A}_{i}(t) \rho(0) \mathbf{A}_{i}^{\dagger}(t)
$$


where the Kraus operators $\left\{\mathbf{A}_{i}\right\}$ have matrix elements given by:

$$
\left[\mathbf{A}_{i}\right]_{m n}(t)=\sqrt{\nu}\langle m|\langle\mu|\mathbf{U}(t)| \nu\rangle| n\rangle ; \quad i=(\mu, \nu) .
$$

Here $|\nu, \mu\rangle$ are basis elements of the bath Hilbert space, and $K=N_{B}^{2}$, where $N_{B}$ is the number of bath degrees of freedom. Also, by unitarity of $\mathbf{U}$, one derives the normalization condition

$$
\sum_{i=0}^{K} \mathbf{A}_{i}^{\dagger} \mathbf{A}_{i}=\mathbf{I}
$$

which guarantees preservation of the trace of $\rho$ :

$$
\operatorname{Tr}[\rho(t)]=\operatorname{Tr}\left[\sum_{i} \mathbf{A}_{i} \rho(0) \mathbf{A}_{i}^{\dagger}\right]=\operatorname{Tr}\left[\rho(0) \sum_{i} \mathbf{A}_{i}^{\dagger} \mathbf{A}_{i}\right]=\operatorname{Tr}[\rho(0)] .
$$

The Kraus operators belong to the Hilbert-Schmidt space $\mathcal{A}(\mathcal{H})$ (itself a Hilbert space) of bounded operators acting on the system Hilbert space, and are represented by $N \times N$ matrices, just like $\rho$. $\mathcal{A}(\mathcal{H})$ is endowed with the scalar product

$$
\left\langle\mathbf{A}_{i}, \mathbf{A}_{j}\right\rangle=\operatorname{Tr}\left[\mathbf{A}_{i} \mathbf{A}_{j}^{\dagger}\right]
$$

\section{B. Fixed-Basis Form of the Operator Sum Representation}

While the OSR evolution equation, Eq. (2.2), is perfectly general, it presents difficulties when trying to separate out the unitary evolution of the system from the possibly non-unitary decoherence which occurs from the coupling of the system to the bath. The reason is that in general, each Kraus operator will contain a contribution from both the unitary and the non-unitary components of the evolution. When one makes the assumption of Markovian dynamics, however, we shall see that the semigroup master equation (SME) does separate the evolution of the system into unitary and non-unitary parts [20]. This motivates us to manipulate the OSR into a form similar to the SME, but without making any Markovian assumption.

It is convenient for this purpose to introduce a fixed operator basis for $\mathcal{A}(\mathcal{H})\left[21\right.$. Let $\left\{\mathbf{K}_{\alpha}\right\}_{\alpha=0}^{M}$, with $\mathbf{K}_{0}=\mathbf{I}$, be such a basis, so that the expansion of the Kraus operators is given by:

$$
\mathbf{A}_{i}(t)=\sum_{\alpha=0}^{M} b_{i \alpha}(t) \mathbf{K}_{\alpha} .
$$

For example, $\left\{\mathbf{K}_{\alpha}\right\}_{\alpha=1}^{M}$ could be the generators of the Lie algebra $s u(N)\left(M=N^{2}-1\right)$, or some sub-algebra thereof (with $M<N^{2}-1$ ) [10]. Under this expansion, the OSR evolution equation, Eq. (2.2), becomes

$$
\rho(t)=\sum_{\alpha, \beta=0}^{M} \chi_{\alpha \beta}(t) \mathbf{K}_{\alpha} \rho(0) \mathbf{K}_{\beta}^{\dagger},
$$

where $\chi_{\alpha \beta}(t)$ is the hermitian matrix

$$
\chi_{\alpha \beta}(t)=\sum_{i=0}^{K} b_{i \alpha}(t) b_{i \beta}^{*}(t) .
$$

Likewise the normalization condition, Eq. (2.4), is given by,

$$
\sum_{\alpha, \beta=0}^{M} \chi_{\alpha \beta}(t) \mathbf{K}_{\beta}^{\dagger} \mathbf{K}_{\alpha}=\mathbf{I}
$$

Next we separate out the action of the identity on both Eq. (2.8) and Eq. (2.10) yielding: 


$$
\begin{gathered}
\rho(t)=\chi_{00} \rho(0)+\sum_{\alpha=1}^{M}\left[\chi_{\alpha 0}(t) \mathbf{K}_{\alpha} \rho(0)+\chi_{0 \alpha}(t) \rho(0) \mathbf{K}_{\alpha}^{\dagger}\right]+\sum_{\alpha, \beta=1}^{M} \chi_{\alpha \beta}(t) \mathbf{K}_{\alpha} \rho(0) \mathbf{K}_{\beta}^{\dagger}, \\
\chi_{00} \mathbf{I}+\sum_{\alpha=1}^{M}\left[\chi_{0 \alpha}(t) \mathbf{K}_{\alpha}^{\dagger}+\chi_{\alpha 0}(t) \mathbf{K}_{\alpha}\right]+\sum_{\alpha, \beta=1}^{M} \chi_{\alpha \beta}(t) \mathbf{K}_{\beta}^{\dagger} \mathbf{K}_{\alpha}=\mathbf{I} .
\end{gathered}
$$

Multiplying Eq. (2.12) by $\frac{1}{2} \rho(0)$ separately from both the left and the right, adding the resulting equations, and substituting the resulting expression for $\chi_{00} \rho(0)$ into Eq. (2.11), we find:

$$
\rho(t)-\rho(0)=-\frac{i}{\hbar}[\mathbf{S}(t), \rho(0)]+\frac{1}{2} \sum_{\alpha, \beta=1}^{M} \chi_{\alpha \beta}(t)\left(\left[\mathbf{K}_{\alpha}, \rho(0) \mathbf{K}_{\beta}^{\dagger}\right]+\left[\mathbf{K}_{\alpha} \rho(0), \mathbf{K}_{\beta}^{\dagger}\right]\right),
$$

where $\mathbf{S}(t)$ is the hermitian operator defined by

$$
\mathbf{S}(t)=\frac{i \hbar}{2} \sum_{\alpha=1}^{M}\left[\chi_{\alpha 0}(t) \mathbf{K}_{\alpha}-\chi_{0 \alpha}(t) \mathbf{K}_{\alpha}^{\dagger}\right]
$$

Eq. (2.13) is the desired result: it represents a fixed-basis OSR evolution equation. This generally resembles the SME in form, but this resemblance should be considered with caution: it can be shown by explicit resummation that for purely unitary evolution, $\rho(t)=\mathbf{U}(t) \rho(0) \mathbf{U}^{\dagger}(t)$, the terms in the fixed-basis OSR are $[\mathbf{S}(t), \rho(0)]=\left[\sin \left(\frac{\mathbf{H} t}{\hbar}\right), \rho(0)\right]$ and $\frac{1}{2} \sum_{\alpha \beta}(\cdots)=\mathbf{U}(t) \rho(0) \mathbf{U}^{\dagger}(t)+(i / \hbar)\left[\sin \left(\frac{\mathbf{H} t}{\hbar}\right), \rho(0)\right]-\rho(0)$. Hence the first term alone $[\mathbf{S}(t), \rho(0)]$ does not necessarily account for the entire unitary dynamics, as one might naively be led to suspect.

\section{Comparison of the Fixed-Basis Operator Sum Representation Equation with the Semigroup Master Equation}

We recall that in the semigroup approach, under the assumptions of (i) Markovian dynamics, (ii) complete positivity, and (iii) initial decoupling between the system and the bath, the system evolves according to the SME [14.20.22]:

$$
\begin{aligned}
\frac{\partial \rho(t)}{\partial t} & =\mathrm{L}[\rho(t)] \equiv-\frac{i}{\hbar}[\mathbf{H}, \rho(t)]+\mathrm{L}_{D}[\rho(t)] \\
\mathrm{L}_{D}[\rho(t)] & =\frac{1}{2} \sum_{\alpha, \beta=1}^{M} a_{\alpha \beta}\left(\left[\mathbf{F}_{\alpha}, \rho(t) \mathbf{F}_{\beta}^{\dagger}\right]+\left[\mathbf{F}_{\alpha} \rho(t), \mathbf{F}_{\beta}^{\dagger}\right]\right),
\end{aligned}
$$

where $a_{\alpha \beta}$ is a constant hermitian matrix. This equation bears a clear resemblance to Eq. (2.13). In fact, taking the derivative of Eq. (2.13), we find

$$
\frac{\partial \rho(t)}{\partial t}=-\frac{i}{\hbar}[\dot{\mathbf{S}}(t), \rho(0)]+\frac{1}{2} \sum_{\alpha, \beta=1}^{M} \dot{\chi}_{\alpha \beta}(t)\left(\left[\mathbf{K}_{\alpha}, \rho(0) \mathbf{K}_{\beta}^{\dagger}\right]+\left[\mathbf{K}_{\alpha} \rho(0), \mathbf{K}_{\beta}^{\dagger}\right]\right) .
$$

Noticing the subtle and important differences between the SME Eq. (2.16) and this OSR evolution equation (2.17) allows us to understand the exact manner in which the semigroup evolution can arises from the OSR evolution under the above mentioned conditions. The most important difference between these two equations is the fact that the SME provides a prescription for determining $\rho(t)$ at all times $t$, given $\rho\left(t^{\prime}\right)$ at any other time $t^{\prime} \geq 0$, whereas Eq. (2.17) determines $\rho(t)$ in terms of $\rho(0)$, i.e., at the special time $t=0$ where the system and the bath are in a product state.

We now show that explicit use of a coarse-graining over time, together with the above-mentioned assumptions, leads one naturally from the OSR evolution equation, Eq. (2.17) to the SME. We note that it is of course possible to derive the SME with other methods, (such as adding an infinite bath [23,24]) given the appropriate assumptions. Our goal here however is not so much to rederive the SME, as to specifically establish a route from the non-Markovian OSR to the Markovian SME.

At this point it is useful to introduce a time-scale $\tau$ for the bath "memory" (whose definition will be made more precise below) and to coarse-grain the evolution of the system in terms of this time scale: 


$$
\rho_{j}=\rho(j \tau) ; \quad \chi_{\alpha \beta ; j}=\chi_{\alpha \beta}(j \tau) ; \quad j \in \mathbf{N} .
$$

Further, rewriting the OSR Eq. (2.13) as $\rho(t)=\boldsymbol{\Lambda}(t) \rho(0)$ and defining $\tilde{\mathrm{L}}(t)$ through $\boldsymbol{\Lambda}(t)=\mathrm{T} \exp \left[\int_{0}^{t} \tilde{\mathrm{L}}(s) d s\right]$ we find that

$$
\frac{\partial \rho(t)}{\partial t}=\tilde{\mathrm{L}}(t)[\rho(t)]
$$

Defining $\tilde{\mathrm{L}}_{j}=\int_{j \tau}^{(j+1) \tau} \tilde{\mathrm{L}}(s) d s$, with $\tau n=t$, we have

$$
\int_{0}^{t} \tilde{\mathrm{L}}(s) d s=\tau \sum_{j=0}^{n-1} \tilde{\mathrm{L}}_{j}
$$

Next we will make the assumption that on the time scale of the bath $\tau$, the evolution generators $\tilde{\mathrm{L}}(t)$ commute in the "average" sense that $\left[\tilde{\mathrm{L}}_{j}, \tilde{\mathrm{L}}_{k}\right]=0, \forall j, k$. Physically, we imagine this operation as arising from the "resetting" of the bath density operator over the time-scale $\tau$. Under this assumption, the evolution of the system is Markovian when $t \gg \tau$ :

$$
\mathbf{\Lambda}(t)=\prod_{j=0}^{n-1} \exp \left[\tau \tilde{\mathrm{L}}_{j}\right]
$$

Further, under the discretization of the evolution, this product form of the evolution implies that

$$
\rho_{j+1}=\exp \left[\tau \tilde{\mathrm{L}}_{j}\right]\left[\rho_{j}\right] .
$$

In the limit of $\tau \ll t$ we expand this exponential, to find that

$$
\frac{\rho_{j+1}-\rho_{j}}{\tau}=\tilde{\mathrm{L}}_{j}\left[\rho_{j}\right]
$$

This equation is simply a discretization of Eq. (2.19) under the assumption that $\tau \ll \theta$, where $\theta$ is the time-scale of change for the system density matrix. Notice in particular that the RHS of Eq. (2.23) contains the average value of $\tilde{\mathrm{L}}(t)$ over the interval. Now, from the OSR evolution equation (2.17), we know the explicit form of $\tilde{\mathrm{L}}(t)$ over the first interval from 0 to $\tau$. Discretizing over this interval we find that

$$
\frac{\rho_{1}-\rho_{0}}{\tau}=-\frac{i}{\hbar}\left[\langle\dot{\mathbf{S}}\rangle, \rho_{0}\right]+\frac{1}{2} \sum_{\alpha, \beta=1}^{M}\left\langle\dot{\chi}_{\alpha \beta}\right\rangle\left(\left[\mathbf{K}_{\alpha}, \rho_{0} \mathbf{K}_{\beta}^{\dagger}\right]+\left[\mathbf{K}_{\alpha} \rho(0), \mathbf{K}_{\beta}^{\dagger}\right]\right) \equiv \tilde{\mathrm{L}}_{0}\left[\rho_{0}\right],
$$

where

$$
\langle X\rangle \equiv \frac{1}{\tau} \int_{0}^{\tau} X(s) d s
$$

Thus, in the sense of the coarse graining above we have arrived at an explicit form for $\tilde{\mathrm{L}}_{0}$. Consider the evolution beyond this first interval. Deriving an explicit form for $\tilde{\mathrm{L}}_{1}$ and for higher terms is now impossible because Eq. (2.17) gives the evolution in terms of $\rho(0)$. However, since we have made the assumption that the bath "resets" over the time-scale $\tau$, we expect the bath to interact with the system in the same manner over every $\tau$-length coarse-grained interval. This is equivalent to assuming that $\tilde{\mathrm{L}}_{i}=\tilde{\mathrm{L}}_{0}, \forall i$ (which of course is the most trivial way of satisfying the Markovian evolution condition $\left[\tilde{\mathrm{L}}_{i}, \tilde{\mathrm{L}}_{j}\right]=0, \forall i, j$ ). Then, using Eq. (2.23), one is led to the well known form of the semigroup equation of motion:

$$
\frac{\partial \rho(t)}{\partial t}=-\frac{i}{\hbar}[\langle\dot{\mathbf{S}}\rangle, \rho(t)]+\frac{1}{2} \sum_{\alpha, \beta=1}^{M}\left\langle\dot{\chi}_{\alpha \beta}\right\rangle\left(\left[\mathbf{K}_{\alpha}, \rho(t) \mathbf{K}_{\beta}^{\dagger}\right]+\left[\mathbf{K}_{\alpha} \rho(t), \mathbf{K}_{\beta}^{\dagger}\right]\right)
$$

(under the natural identification of the K's with the F's of the SME).

We can write this equation of motion in an alternative form which distinguishes between the system and bath contributions to the Liouvillian evolution. Because Eq. (2.17) is linear in the $\chi_{\alpha \beta}(t)$ matrix, one can calculate $\chi_{\alpha \beta}^{(0)}(t)$ 
for the isolated system and hence define the new terms which come about from the coupling of the system to the bath:

$$
\chi_{\alpha \beta}(t)=\chi_{\alpha \beta}^{(0)}(t)+\chi_{\alpha \beta}^{(1)}(t) .
$$

The terms which correspond to the isolated system will therefore produce a normal $-(i / \hbar)[\mathbf{H}, \rho(t)]$ Liouville term in Eq. (2.26). Thus Eq. (2.26) can be rewritten as

$$
\frac{\partial \rho(t)}{\partial t}=-\frac{i}{\hbar}\left[\mathbf{H}+\left\langle\dot{\mathbf{S}}^{(1)}\right\rangle, \rho(t)\right]+\frac{1}{2} \sum_{\alpha, \beta=1}^{M}\left\langle\dot{\chi}_{\alpha \beta}^{(1)}\right\rangle\left(\left[\mathbf{K}_{\alpha}, \rho(t) \mathbf{K}_{\beta}^{\dagger}\right]+\left[\mathbf{K}_{\alpha} \rho(t), \mathbf{K}_{\beta}^{\dagger}\right]\right)
$$

which with the identification of $\left\langle\dot{\chi}_{\alpha \beta}\right\rangle$ with $a_{\alpha \beta}$, and $\mathbf{K}_{\alpha}$ with $\mathbf{F}_{\alpha}$, is equivalent to Eqs. 2.15)-(2.16), except for the presence of the second term derived from $\left\langle\dot{\mathbf{S}}^{(1)}\right\rangle$ in the Liouvillian. This second term inducing unitary dynamics on the system, $\left\langle\dot{\mathbf{S}}^{(1)}\right\rangle$, is referred to as the Lamb shift. It explicitly describes the effect the bath has on the unitary part of the system dynamics and "renormalizes" the system Hamiltonian. It is often implicitly assumed to be present in Eq. (2.15) [25].

In summary, we have shown in this Section how coarse-graining the evolution over the bath time-scale $\tau$ allows one to understand the connection between the OSR and the semigroup evolution. Specifically, we have made the assumptions that (i) the time-scale for the evolution of the system density matrix is much larger than the time-scale for the resetting of the bath $(\tau \gg \theta)$, (ii) the evolution of the system should be Markovian $\left(\left[\tilde{\mathrm{L}}_{i}, \tilde{\mathrm{L}}_{j}\right]=0, \forall i, j\right)$, and (iii) the bath resets to the same state so that the system evolution is the same over every coarse graining $\left(\tilde{\mathrm{L}}_{i}=\tilde{\mathrm{L}}_{0}, \forall i\right)$. This last assumption can be relaxed, and replaced by an ensemble average taken over the different states to which the bath resets, i.e. $\tilde{\mathrm{L}}_{i}=\left\langle\left\langle\tilde{\mathrm{L}}_{i}\right\rangle\right\rangle$. The importance of Eq. (2.17) lies in the fact that it allows one to pinpoint the exact point at which the assumption of Markovian dynamics are made and further, due to the general likeness of its form to the SME, provides an easily translatable connection when going from the non-Markovian OSR to the Markovian SME. Notice also that the assumption of Markovian dynamics introduces an arrow of time in the evolution of the system through the ordering of the environmental states: the system evolves through time in the direction of each successive resetting of the bath.

\section{SHORT-TIME EXPANSIONS OF THE MEMORY FIDELITY IN THE OSR}

The mixed-state memory fidelity [26]

$$
F_{\mathrm{m}}(t)=\operatorname{Tr}[\rho(0) \rho(t)]
$$

is a good measure of the degree to which a system serves as a perfect quantum memory. $F_{\mathrm{m}}(t)$ is the mixed state analog of the survival probability for a pure state wavefunction. When the initial preparation is pure, a perfect, noiseless quantum memory will have $F_{\mathrm{m}}(t)=1$, but in the noisy case $0 \leq F_{\mathrm{m}}(t) \leq 1$. If one starts out in a mixed state then $F_{\mathrm{m}}(0)<1$, and it is usually necessary to resort to some kind of purification [27]. We will consider here only short-time expansions of the fidelity, since it is known that using QECC it is possible to restore the coherence of a quantum system as long as corrections are applied sufficiently frequently [16]. Thus, we perform a power expansion of the fidelity in time [28]

$$
F_{\mathrm{m}}(t)=\sum_{n} \frac{1}{n !}\left(\frac{t}{\tau_{n}}\right)^{n}
$$

where the decoherence rates are defined as

$$
\frac{1}{\tau_{n}}=\left\{\operatorname{Tr}\left[\rho(0) \rho^{(n)}(0)\right]\right\}^{1 / n},
$$

and $\rho^{(n)}$ denotes the $n^{\text {th }}$ time derivative of the density matrix. 


\section{A. First Order Decoherence Rate in the OSR}

Throughout the literature on decoherence there abound many examples of non-zero first order decoherence rates (e.g. [9,29,30 ). Specific attention has been given to maximizing this time scale in order to maintain long-lived coherent states. We therefore pose the question here, how do the first order decoherence rates for non-Markovian evolution behave within the OSR? The first order decoherence rate is given by

$$
\frac{1}{\tau_{1}}=\operatorname{Tr}[\rho(0) \dot{\rho}(0)]
$$

We note that by substituting in the reduced density matrix, Eq. (2.1), and evaluating the derivative at $t=0$, we are immediately led to the vanishing of the first order decoherence rate from the cyclic property of the trace:

$$
\frac{1}{\tau_{1}}=\operatorname{Tr}_{S}\left[\rho(0) \operatorname{Tr}_{B}\left[-i \mathbf{H}_{S B} \rho(0)+i \rho(0) \mathbf{H}_{S B}\right]\right]=0
$$

Therefore for the general non-Markovian dynamics, first order decoherence rates are rigorously zero, provided that $\mathbf{H}_{S B}$ is finite. What is not obvious from this simple manipulation is how a coarse-graining procedure can lead to the commonly encountered non-vanishing first order decoherence rates. This therefore suggests employing the "pre-coarsegrained" OSR, Eq. (2.17), for the derivative, and then carrying out the specific coarse-graining procedure outlined in the previous section on Eq. (3.4), in order to understand how non-vanishing first order decoherence rates can arise.

Using Eq. (2.17) the first order decoherence rate becomes

$$
\frac{1}{\tau_{1}}=\operatorname{Tr}\left[\rho(0)\left(-\frac{i}{\hbar}[\dot{\mathbf{S}}(0), \rho(0)]+\frac{1}{2} \sum_{\alpha, \beta=1}^{M} \dot{\chi}_{\alpha \beta}(0)\left(\left[\mathbf{K}_{\alpha}, \rho(0) \mathbf{K}_{\beta}^{\dagger}\right]+\left[\mathbf{K}_{\alpha} \rho(0), \mathbf{K}_{\beta}^{\dagger}\right]\right)\right)\right] .
$$

Using the decomposition of the Kraus operators, Eq. (2.2), and knowing that $\mathbf{U}(0)=\mathbf{I}_{S} \otimes \mathbf{I}_{B}$, we find that $\mathbf{A}_{i}(0)=$ $\sqrt{\nu} \mathbf{I}_{S} \delta_{i,(\nu, \nu)}$. Thus, since the $\mathbf{K}_{\alpha}$ 's form a linearly independent basis, it follows, using Eq. (2.7), that the expansion coefficients must be

$$
b_{i \alpha}(0)=\delta_{\alpha 0} \sqrt{\nu} \delta_{i,(\nu, \nu)}
$$

By direct evaluation,

$$
\dot{\chi}_{\alpha \beta}(0)=\sum_{\nu} \sqrt{\nu}\left[\left(\delta_{\alpha 0} \dot{b}_{(\nu, \nu), \beta}^{*}(0)+\delta_{\beta 0} \dot{b}_{(\nu, \nu), \alpha}(0)\right)\right]
$$

which implies the vanishing [as long as $\dot{b}_{(\nu, \nu), \alpha}(0)$ remains finite] in Eq. 3.6) of every term except $\operatorname{Tr}[\rho(0)[\dot{\mathbf{S}}(0), \rho(0)]]$. However, this in turn vanishes by cyclic permutation of the trace. Thus we see that within the OSR, the first order decoherence rate is always zero when the $\dot{b}_{(\nu, \nu), \alpha}(0)$ remain finite. To determine the significance of this restriction we choose as a basis for the Kraus operators a set of $M \mathbf{K}_{\alpha}$ 's which form a Lie algebra and hence have a suitably defined inner product $[\mathrm{Eq} \cdot(2.6)]$ :

$$
\operatorname{Tr}\left[\mathbf{K}_{\alpha} \mathbf{K}_{\beta}^{\dagger}\right]=\left\{\begin{array}{ccc}
\delta_{\alpha \beta} & \text { for } & \alpha \geq 1, \beta \geq 1 \\
N \delta_{\beta 0} \delta_{\alpha 0} & \text { otherwise }
\end{array}\right.
$$

We then find using the definition of the Kraus operators, Eq. (2.3),

$$
b_{(\nu \nu), \alpha}(t)=\operatorname{Tr}\left[\mathbf{K}_{\alpha}^{\dagger} \sqrt{\nu}\langle\nu|\mathbf{U}(t)| \nu\rangle\right] .
$$

Differentiating this and recalling that $\mathbf{U}(t)=\exp \left(-i \mathbf{H}_{S B} t / \hbar\right)$, we find that

$$
\dot{b}_{(\nu \nu), \alpha}(0)=-\frac{i}{\hbar} \operatorname{Tr}\left[\mathbf{K}_{\alpha}^{\dagger} \sqrt{\nu}\left\langle\nu\left|\mathbf{H}_{S B}(0)\right| \nu\right\rangle\right] .
$$

Thus in order for $\dot{b}_{(\nu \nu), \alpha}(0)$ to remain finite, $\left\langle\nu\left|\mathbf{H}_{S B}(0)\right| \nu\right\rangle$ must be finite. Hence our conclusion that the first order decoherence rate vanishes in the OSR is valid for any finite total Hamiltonian (by which we mean its matrix elements), and conversely, any finite total Hamiltonian will have zero first order decoherence rate [31. 
Examination of our derivation of the SME, Eqs. $(2.26)$ and $(2.28)$, now shows how non-zero first order decoherence rates can arise when the evolution is considered to be Markovian. In the derivation of the semigroup equation in the Markovian limit we made the assumption that the matrices $\dot{\chi}_{\alpha \beta}(t)$ can be identified with the constant matrices $a_{\alpha \beta}$ of the semigroup equation, Eq. (2.16). However, when this is done, the matrix elements $\dot{\chi}_{\alpha \beta}(0)$ in Eq. (3.6) are replaced by their time-averaged values, for which the relation (3.8) no longer applies. Hence, in general, the first order decoherence rates are necessarily not zero when the Markovian coarse-graining is applied. For a finite total Hamiltonian $\mathbf{H}_{S B}$, non-zero first order rates are therefore seen to be an artifact of the Markovian assumption, and their appearance emphasizes the delicate nature of the transition to the Markovian regime.

\section{B. Example: Phase Damping}

The restriction to a finite total Hamiltonian above may at first sight seem obvious. However, consider, for instance, the often quoted example of phase damping of a qubit. In this case, it would appear that there is a finite first order decoherence rate. Yet, it is often presented within the OSR [32.33], which, as we have just shown above, would predict zero first order decoherence rates for any non-singular Hamiltonian. In this example, the Kraus operators are given by [32]

$$
\mathbf{A}_{0}=\left(\begin{array}{cc}
1 & 0 \\
0 & e^{-\lambda t}
\end{array}\right) \quad \mathbf{A}_{1}=\left(\begin{array}{cc}
0 & 0 \\
0 & \sqrt{1-e^{-2 \lambda t}}
\end{array}\right)
$$

and a simple calculation using these operators yields a minimum first order decoherence rate of $1 / \tau_{1}=-\lambda / 2$. How can this be?

To resolve this dichotomy, we consider how the above phase damping Kraus operators are generated from the unitary dynamics of a qubit system $S$ and a qubit bath $B$. The evolution operator

$$
\mathbf{U}(t)=\left(\begin{array}{cccc}
1 & 0 & 0 & 0 \\
0 & e^{-\lambda t} & 0 & \sqrt{1-e^{-2 \lambda t}} \\
0 & 0 & 1 & 0 \\
0 & -\sqrt{1-e^{-2 \lambda t}} & 0 & e^{-\lambda t}
\end{array}\right) \begin{aligned}
& |\downarrow 0\rangle \\
& |\downarrow 1\rangle \\
& |\uparrow 0\rangle \\
& |\uparrow 1\rangle
\end{aligned}
$$

[where the first qubit represents the bath $(|\uparrow\rangle,|\downarrow\rangle)$ and the second represents the system $(|0\rangle,|1\rangle)$ as denoted in the columns above] with the bath initially in the state $|\downarrow\rangle$, immediately gives the Kraus operators of Eq. (3.12). Now, it is easy to calculate the Hamiltonian which generates this evolution, [using $\mathbf{H}_{S B}(t)=i \hbar \frac{d \mathbf{U}(t)}{d t} \mathbf{U}^{\dagger}(t)$ ]:

$$
\mathbf{H}_{S B}(t)=\left(\begin{array}{cccc}
0 & 0 & 0 & 0 \\
0 & 0 & 0 & -g(t) \\
0 & 0 & 0 & 0 \\
0 & g(t) & 0 & 0
\end{array}\right)
$$

where

$$
g(t)=i \hbar \frac{\gamma e^{-\gamma t}}{\sqrt{1-e^{-2 \gamma t}}}
$$

However, we see that as $t \rightarrow 0, g(t) \rightarrow \infty$. Thus, in this simple example, we find that at $t=0$, the Hamiltonian becomes singular. This illustrates our claim that first order decoherence rates in the OSR are the result of an infinite Hamiltonian, and do not contradict the general OSR result of zero rates for finite Hamiltonians.

The diverging Hamiltonian in this example is in fact equivalent to non-closedness of the system $S+B$. It is well known that phase damping can be generated by a model of random phase-kicks [34], which implies an external random force, i.e., that the system $S+B$ is in fact not closed. Since this is in contradiction to our initial assumptions (Sec. II A), it should not come as a surprise that a non-vanishing first order decoherence rate is found in this situation. A similar divergence will result of course from a bath with an infinite number of degrees of freedom [23,24. The OSR phase damping example thus can still be used (as is commonly done in the analysis of quantum error correction) under the caveat that one cannot claim that it arises from a finite closed system $(\mathrm{S}+\mathrm{B})$. 


\section{EFFECT OF SYMMETRY BREAKING PERTURBATIONS ON MEMORY FIDELITY OF DECOHERENCE-FREE SUBSPACES}

Our discussion in the previous sections was completely general, dealing with the decoherence of the entire system Hilbert space. We now restrict our attention to the behavior of the fidelity in DF subspaces. We first briefly summarize the basic theory of DF subspaces and then generalize the first order stability results obtained within the SME in Ref. [10].

\section{A. Theory of Decoherence-Free Subspaces: Markovian vs non-Markovian Approach}

Recently, conditions for the existence of decoherence free subspaces within the framework of the Markovian SME approach [9,10] and in a non-Markovian [7] setting were derived. We first clarify here the connection between the SME and the non-Markovian results.

In the SME approach it was shown that a necessary and sufficient condition for decoherence free dynamics $\left(\mathrm{L}_{D}[\tilde{\rho}(t)]=0\right)$ in a subspace $\tilde{\mathcal{H}}=\operatorname{Span}\left[\left\{|\tilde{\imath}\rangle_{i=1}^{N_{0}}\right\}\right]$ is that all of the basis states $|\tilde{\imath}\rangle$ satisfy the condition

$$
\mathbf{F}_{\alpha}|\tilde{\imath}\rangle=c_{\alpha}|\tilde{\imath}\rangle \quad \forall \alpha, \tilde{\imath}
$$

where the $\mathbf{F}_{\alpha}$ 's are the error generators in the semigroup Eq. (2.16). Since the $\left\{\mathbf{F}_{\alpha}\right\}$ form a Lie algebra $\mathcal{L}$, this condition has a simple group-theoretic interpretation, namely, the DF states are the singlets of $\mathcal{L}$, i.e., they are the states that transform according to the 1-dimensional representations of $\mathcal{L}$. From Sec. [IIC, and in particular Eq. (2.26), it follows that these error generators become identical to the $\left\{\mathbf{K}_{\alpha}\right\}$ (the basis operators in the fixed expansion of the Kraus operators) when the short-time averaging approximations leading from the OSR to the SME are made.

Within the framework of non-Markovian evolution, it has likewise been shown [7, 11] that a necessary and sufficient condition for decoherence free dynamics over a similar subspace $\tilde{\mathcal{H}}=\operatorname{Span}\left[\left\{|\tilde{\imath}\rangle_{i=1}^{N_{0}}\right\}\right]$ is that all of the basis states $|\tilde{\imath}\rangle$ satisfy the condition

$$
\mathbf{S}_{\alpha}|\tilde{\imath}\rangle=c_{\alpha}|\tilde{\imath}\rangle \quad \forall \alpha, \tilde{\imath}
$$

where $\mathbf{S}_{\alpha}$ 's (system operators) are defined by the interaction Hamiltonian

$$
\mathbf{H}_{I}=\sum_{\alpha} \mathbf{S}_{\alpha} \otimes \mathbf{B}_{\alpha} .
$$

The $\mathbf{B}_{\alpha}$ are bath operators. The fixed-basis OSR equation (2.13) sheds light on the relationship between these two DF conditions. To the extent that the error generators $\mathbf{F}_{\alpha}$ can be derived from expansion of the Kraus operators with a subsequent short-time averaging approximation, the DF condition given for the non-Markovian dynamics are more general than that given by the semigroup approach. We notice that in the limit of small averaging time $\tau$, the error generators $\mathbf{F}_{\alpha}$ 's will, in fact, correspond directly to the $\mathbf{S}_{\alpha}$ 's. This can be seen by expanding the full evolution operator $\mathbf{U}(t)$ to first order in $\tau$ :

$$
\mathbf{U}(\tau)=\mathbf{I}-\frac{i}{\hbar} \mathbf{H}_{S B} \tau+O\left(\tau^{2}\right) .
$$

To this order, the Kraus operators $\mathbf{A}_{i}(t)$ [see Eq. (2.3 )] will only contain terms which correspond to terms that appear in the Hamiltonian $\mathbf{H}_{S B}$. These are $\mathbf{H}$, the system Hamiltonian; $\mathbf{I}$, the identity; and the $\mathbf{S}_{\alpha}{ }^{\prime} \mathrm{s}$ from Eq. (4.3). Terms corresponding to $\mathbf{H}$ will result in unitary evolution of the system, while $\mathbf{I}$ is removed from the set of Kraus operators (since these are considered error generators) via our derivation of the SME. To first order in time, therefore the only error generators are the $\mathbf{S}_{\alpha}$ 's. Thus in the case of small averaging time $\tau$ we see that the two DF conditions are exactly equivalent.

However, it is important to note that the SME approach has other advantages. Thus in many cases it is either impractical or undesirable to derive the $\left\{\mathbf{F}_{\alpha}\right\}$ from a short-time expansion of the type discussed in Sec. II G. In fact, in Lindblad's axiomatic approach [14], the $\left\{\mathbf{F}_{\alpha}\right\}$ are the primary objects and they do not follow from an expansion of a unitary operator. While the $\left\{\overline{\mathbf{F}}_{\alpha}\right\}$ are often identified heuristically from a factorization such as in Eq. (4.3) [35], in some cases (notably strong coupling) one simply cannot clearly separate system and bath in the form assumed in that equation. In this sense, then, the SME provides greater generality than the non-Markovian approach within the Hamiltonian representation subject to Eq. (4.3). Motivated by this aspect, we take condition (4.1) to be necessary and sufficient for DF subspaces. 
Further, one should note that while the semigroup DF condition, Eq. (4.1), guarantees that the evolution of the system will be unitary, the system may still be subject to unitary evolution induced by the bath in the form of the Lamb shift. Such bath-induced evolution, although it does not introduce decoherence, is undesirable in the course of a quantum computation. To the extent that we desire the DF subspace to serve as the basis for a quantum computer, we therefore must impose one of two conditions on the DF subspace. These conditions are: (1) suitable control over the system is obtained so that the Lamb shift term can be canceled out, or, (2) the Lamb shift does not induce dynamics on the subspace, i.e., $\mathbf{H}_{\mathrm{Lamb}}|\tilde{\imath}\rangle=h_{l}|\tilde{\imath}\rangle$. Under the first condition, the DF subspace is not reduced in dimension. However this condition may be physically impossible to realize. The second condition does not make any assumptions about the amount of control one has over the system Hamiltonian, but it may have the undesirable effect of causing a reduction in the size of the DF subspace. Similar conclusions regarding the effect of the Lamb shift hold for the non-Markovian case.

Finally, in both the SME representation and the OSR, the implicit assumption has been made that the system Hamiltonian does not induce the evolution of states from within DF subspace to states outside of the DF subspace:

$$
\mathbf{H}|\tilde{\imath}\rangle=\sum_{j} h_{i j}|\tilde{j}\rangle .
$$

It is a simple matter to satisfy this additional condition in the SME. However, this is not the case in the OSR due to the absence of an explicit appearance of $\mathbf{H}$.

We now proceed to the question of stability of DF subspaces in the non-Markovian and Markovian cases. The calculations in the next two subsections are rather tedious, and the reader who is not interested in the details may wish to skip directly to the results for the memory fidelity, summarized in Table I.

\section{B. Stability of the Memory Fidelity in the Non-Markovian Case}

Consider the addition to a DF subspace of new perturbing terms in the interaction Hamiltonian: $\mathbf{H}_{S B}^{\prime}=\mathbf{H}_{S B}+\epsilon \mathbf{H}_{I}^{\prime}$. In this case, we find that to first order in $\epsilon$, the new full evolution operator is given by

$$
\begin{aligned}
\mathbf{U}^{\prime}(t) & =\sum_{n=0}^{\infty} \frac{(-i t / \hbar)^{n}}{n !}\left(\mathbf{H}_{S B}+\epsilon \mathbf{H}_{I}^{\prime}\right)^{n} \\
& =\mathbf{U}(t)+\sum_{k=1}^{\infty} \epsilon^{k} \sum_{n=k}^{\infty} \frac{(-i t / \hbar)^{n}}{n !} f_{n}^{(k)}\left(\mathbf{H}_{S B}, \mathbf{H}_{I}^{\prime}\right),
\end{aligned}
$$

where

$$
\begin{aligned}
f_{1}^{(1)}\left(\mathbf{H}_{S B}, \mathbf{H}_{I}^{\prime}\right) & =\mathbf{H}_{I}^{\prime} \\
f_{2}^{(1)}\left(\mathbf{H}_{S B}, \mathbf{H}_{I}^{\prime}\right) & =\mathbf{H}_{S B} \mathbf{H}_{I}^{\prime}+\mathbf{H}_{I}^{\prime} \mathbf{H}_{S B} \\
f_{3}^{(1)}\left(\mathbf{H}_{S B}, \mathbf{H}_{I}^{\prime}\right) & =\mathbf{H}_{S B}^{2} \mathbf{H}_{I}^{\prime}+\mathbf{H}_{S B} \mathbf{H}_{I}^{\prime} \mathbf{H}_{S B}+\mathbf{H}_{I}^{\prime} \mathbf{H}_{S B}^{2} \\
f_{2}^{(2)}\left(\mathbf{H}_{S B}, \mathbf{H}_{I}^{\prime}\right) & =\mathbf{H}_{I}^{\prime 2} \\
f_{3}^{(2)}\left(\mathbf{H}_{S B}, \mathbf{H}_{I}^{\prime}\right) & =\mathbf{H}_{S B} \mathbf{H}_{I}^{\prime 2}+\mathbf{H}_{I}^{\prime} \mathbf{H}_{S B} \mathbf{H}_{I}^{\prime}+\mathbf{H}_{I}^{\prime 2} \mathbf{H}_{S B},
\end{aligned}
$$

etc. Here $\mathbf{U}(t)$ is the unperturbed evolution operator. From Eqs. (2.3) and (2.7) we thus see that to first order in $\epsilon$ the operators $\mathbf{K}_{\alpha}$ in which the Kraus operators are expanded, will have new terms due to $\left\{f_{n}^{(1)}\right\}$, hereby denoted by $\left\{\epsilon \mathbf{G}_{p}\right\}_{p=1}^{P}$, which are proportional to $\epsilon$. These terms modify the evolution over the DF subspace [Eq. (2.13)] so that $\partial \tilde{\rho} / \partial t \mapsto \partial \tilde{\rho}^{\prime} / \partial t=\partial \tilde{\rho} / \partial t+\mathrm{L}^{\prime}(t)[\tilde{\rho}(0)]$, where:

$$
\begin{aligned}
\mathrm{L}^{\prime}(t)[\tilde{\rho}(0)] \equiv & -\frac{i}{\hbar}\left[\mathbf{S}^{\prime}(t), \tilde{\rho}(0)\right]+\frac{1}{2} \sum_{\alpha=1}^{M} \sum_{p=1}^{P} \chi_{\alpha p}(t) \mathrm{L}_{\mathbf{K}_{\alpha}, \epsilon \mathbf{G}_{p}}[\tilde{\rho}(0)]+\chi_{\alpha p}^{*}(t) \mathrm{L}_{\epsilon} \mathbf{G}_{p}, \mathbf{K}_{\alpha}[\tilde{\rho}(0)] \\
& +O\left(\epsilon^{2}\right) .
\end{aligned}
$$

Here:

$$
\mathbf{S}^{\prime}(t)=\epsilon \frac{i \hbar}{2} \sum_{p=1}^{P}\left[\chi_{p 0}(t) \mathbf{G}_{p}-\chi_{0 p}(t) \mathbf{G}_{p}^{\dagger}\right]
$$


and:

$$
\mathrm{L}_{\mathbf{x}, \mathbf{y}}[\rho] \equiv\left[\mathbf{x}, \rho \mathbf{y}^{\dagger}\right]+\left[\mathbf{x} \rho, \mathbf{y}^{\dagger}\right] .
$$

Terms of $O\left(\epsilon^{2}\right)$, not written out explicitly in Eq. (4.8), include $\mathrm{L}_{\epsilon} \mathbf{G}_{p}, \epsilon \mathbf{G}_{q}[\tilde{\rho}(0)], \mathrm{L}_{\epsilon^{2}} \mathbf{G}_{p}, \mathbf{K}_{\alpha}[\tilde{\rho}(0)]$, etc. Assuming that $\epsilon \ll$ 1 , we may neglect these terms. Now, for the purposes of argument, we will assume that the system has perfect quantum memory over the DF subspace in the absence of the perturbing error generators, i.e., $F_{\mathrm{m}}^{(\mathrm{DF})}(t)=\operatorname{Tr}[\tilde{\rho}(0) \tilde{\rho}(t)]=1$. The perturbation, however, decreases the fidelity below this perfect value. The modified memory fidelity can be written formally as:

$$
F_{\mathrm{m}}^{\prime}(t)=1-\sum_{k=1} \sum_{n=k} \frac{1}{n !} \frac{t^{n}}{\left(\tau_{n}^{(k)}\right)^{n}}
$$

where $\tau_{n}^{(k)}$ represents the $O\left(\epsilon^{k}\right)$ contribution to the $n^{\text {th }}$ order decoherence time $\tau_{n}$. It was shown in Ref. 10 that, within the SME, the term which is first order in both the perturbation and time vanishes: $1 / \tau_{1}^{(1)}=0$. This left open the possibility of terms of order $O\left(\epsilon t^{2}\right)$ and higher spoiling the fidelity. Here we will generalize this result in the non-Markovian case [the Markovian case will be dealt with in Sec. (IV d)] and show that in fact $1 / \tau_{n}^{(1)}=0$ for all $n$, so that the entire $O(\epsilon)$ contribution vanishes, and only terms of order $O\left(\epsilon^{2} t^{2}\right)$ can spoil the memory fidelity. For simplicity of notation, since we are considering here only the $1 / \tau_{n}^{(1)}$ decoherence rates, we drop the $(k)$ superscript from now on.

The perturbed decoherence rates are thus given by:

$$
\left(\frac{1}{\tau_{n}}\right)^{n}=\operatorname{Tr}\left[\tilde{\rho}(0)\left\{\tilde{\rho}^{\prime}(t)\right\}^{(n)}\right]=\operatorname{Tr}\left[\tilde{\rho}(0)\left\{\mathrm{L}^{\prime}(t)[\tilde{\rho}(0)]\right\}_{t=0}^{(n-1)}\right] .
$$

Using Eq. (4.8) and noting that the terms involving $\mathbf{S}^{\prime}$ vanish directly by permutation under the trace, we obtain to first order in $\epsilon$ :

$$
\left(\frac{1}{\tau_{n}}\right)^{n}=\frac{1}{2}\left[\sum_{\alpha=1}^{M} \sum_{p=1}^{P} \chi_{\alpha p}^{(n-1)}(t) \operatorname{Tr}\left[\tilde{\rho}(0) \mathrm{L}_{\mathbf{K}_{\alpha}, \in \mathbf{G}_{p}}[\tilde{\rho}(0)]\right]+\chi_{\alpha p}^{*(n-1)}(t) \operatorname{Tr}\left[\tilde{\rho}(0) \mathrm{L}_{\epsilon} \mathbf{G}_{p}, \mathbf{K}_{\alpha}[\tilde{\rho}(0)]\right]\right] .
$$

To evaluate this, we need to know $\mathbf{K}_{\alpha} \rho \tilde{(0)}$. Now, when we expand the Kraus operators about a fixed basis $\mathbf{K}_{\alpha}$ as in Eq. (2.7), this basis will consist of all possible products of the three terms $\mathbf{H}, \mathbf{S}_{\alpha}$, and $\mathbf{I}$ [recall the definition of the Kraus operators, Eq. (2.3)]. Assuming a perfect quantum memory

$$
[\mathbf{H}, \tilde{\rho}(0)]=0,
$$

we can commute $\mathbf{H}$ with $\tilde{\rho}(0)$ and, using the DF condition $\mathbf{S}_{\alpha}|\tilde{\imath}\rangle=c_{\alpha}|\tilde{\imath}\rangle$, for a given product of $\mathbf{S}_{\alpha}$ 's and $\mathbf{H}$ 's we can replace each $\mathbf{S}_{\alpha}$ with its eigenvalue $c_{\alpha}$. Thus, for example,

$$
\mathbf{H}^{2} \mathbf{S}_{1} \mathbf{H} \tilde{\rho}=\mathbf{H}^{2} \mathbf{S}_{1} \tilde{\rho} \mathbf{H}=\mathbf{H}^{2} c_{1} \tilde{\rho} \mathbf{H}=c_{1} \mathbf{H}^{3} \tilde{\rho} .
$$

It follows that for a DF subspace in the OSR, the basis operators $\mathbf{K}_{\alpha}$ will satisfy the condition

$$
\mathbf{K}_{\alpha} \tilde{\rho}(t)=d_{\alpha} \mathbf{H}^{m_{\alpha}} \tilde{\rho}(t),
$$

with $m_{\alpha}$ an integer and $d_{\alpha}$ a real number. Using this result, we then cycle the trace in Eq. (4.12), and again using $[\mathbf{H}, \tilde{\rho}(0)]=0$, we find for the first trace

$$
\begin{aligned}
& \operatorname{Tr}\left[\tilde{\rho}(0) \mathbf{L}_{\mathbf{K}_{\alpha}, \epsilon \mathbf{G}_{p}}[\tilde{\rho}(0)]\right] \\
= & \epsilon d_{\alpha} \operatorname{Tr}\left[2 \tilde{\rho}(0) \mathbf{H}^{m_{\alpha}} \tilde{\rho}(0) \mathbf{G}_{p}^{\dagger}-\tilde{\rho}(0) \mathbf{G}_{p}^{\dagger} \mathbf{H}^{m_{\alpha}} \tilde{\rho}(0)-\tilde{\rho}(0) \mathbf{G}_{p}^{\dagger} \tilde{\rho}(0) \mathbf{H}^{m_{\alpha}}\right] \\
= & 0 .
\end{aligned}
$$

The second trace vanishes similarly. Thus we see that all orders of decoherence rates must vanish to first order in $\epsilon$, i.e., $1 / \tau_{n}=0$. Examining Eq. (4.8), it is clear that the second order $\epsilon^{2}$ term does not lead to a similar vanishing of the traces. Therefore we have proved that: under non-Markovian evolution, DF subspaces are completely stable to first order under a symmetry breaking perturbation, where by "completely" we mean explicitly stable to all orders of time. 


\section{Stability of the Memory Fidelity in the Markovian Case}

The stability of DF subspaces with respect to symmetry breaking perturbations in the non-Markovian case derived above is an significant extension of the stability derived in Ref. 10 for the Markovian SME. However, the result presented in Ref. [10] only examined the effect of a symmetry breaking perturbation on the first order decoherence rate $\left(1 / \tau_{1}^{(1)}=0\right)$. Here we show that the stronger non-Markovian result derived above $\left(1 / \tau_{n}^{(1)}=0 \forall n \geq 1\right)$ also holds in the Markovian SME.

The effect of perturbing a DF subspace in the SME is the addition of new error generators, hereby denoted by $\left\{\epsilon \mathbf{G}_{p}\right\}_{p=1}^{P}$, to the master equation (which was partially treated in Ref. 10]) as well as a perturbing Lamb shift term in the master equation (which was not treated in Ref. [10]). These terms modify the SME [Eqs. (2.15)-(2.16)] so that $\partial \rho(t) / \partial t=\mathrm{L}^{\prime}[\rho(t)]$, with

$$
\begin{aligned}
\mathrm{L}^{\prime}[\rho(t)] & \equiv \mathrm{L}[\rho(t)]-\frac{i}{\hbar}\left[\epsilon \mathbf{H}_{\mathrm{Lamb}}^{\prime}, \rho(t)\right] \\
& +\frac{1}{2} \sum_{p=1}^{P} \sum_{\alpha=1}^{M}\left(g_{p \alpha} \mathrm{L}_{\epsilon} \mathbf{G}_{p}, \mathbf{F}_{\alpha}[\rho(t)]+g_{p \alpha}^{*} \mathrm{~L}_{\mathbf{F}_{\alpha}, \epsilon \mathbf{G}_{p}}[\rho(t)]\right)+O\left(\epsilon^{2}\right),
\end{aligned}
$$

where $\mathrm{L}[\rho(t)]$ is the unperturbed SME term given by Eq. (2.15), $\epsilon \mathbf{H}_{\mathrm{Lamb}}^{\prime}$ is the perturbing Lamb shift, and $\mathrm{L}_{\mathbf{x}, \mathbf{y}}[\rho(t)]$ is given by Eq. (4.10). The perturbed decoherence rates are given by

$$
\left(\frac{1}{\tau_{n}}\right)^{n}=\operatorname{Tr}\left[\tilde{\rho}(0) \tilde{\rho}^{(n)}(0)\right]=\operatorname{Tr}\left[\tilde{\rho}(0)\left\{\left(\mathrm{L}^{\prime}\right)^{n}[\tilde{\rho}(0)]\right\}\right],
$$

where $\left(\mathrm{L}^{\prime}\right)^{n}[\tilde{\rho}(0)]=\mathrm{L}^{\prime}\left[\mathrm{L}^{\prime}\left[\ldots \mathrm{L}^{\prime}[\tilde{\rho}(0)]\right]\right], n$ times. To evaluate this expression, recall $(\mathrm{i})$ the $\mathrm{DF}$ condition $\mathrm{L}[\tilde{\rho}(0)]=0$ and (ii) that we are working only to first order in $\epsilon$. Now, for simplicity, consider first $\left(\mathrm{L}^{\prime}\right)^{2}[\tilde{\rho}(0)]$, and denote the second and third terms on the RHS of Eq. 4.17) by $\mathrm{A}_{1}[\rho(t)]$ and $\mathrm{A}_{2}[\rho(t)]$. By the DF condition, $\mathrm{L}[\mathrm{L}[\tilde{\rho}(0)]]=\mathrm{A}_{1}[\mathrm{~L}[\tilde{\rho}(0)]]=$

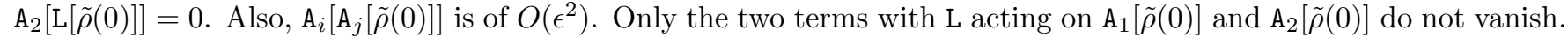
This reasoning generalizes easily for $n>2$, so we find that to first order in $\epsilon$,

$$
\begin{aligned}
& \left(\frac{1}{\tau_{n}^{(1)}}\right)^{n}= \\
& \operatorname{Tr}\left[\tilde{\rho}(0) \mathrm{L}^{n-1}\left[-\frac{i}{\hbar}\left[\epsilon \mathbf{H}_{\mathrm{Lamb}}^{\prime}, \tilde{\rho}(0)\right]+\frac{1}{2} \sum_{p=1}^{P} \sum_{\alpha=1}^{M}\left(g_{p \alpha} \mathrm{L}_{\epsilon} \mathbf{G}_{p}, \mathbf{F}_{\alpha}[\tilde{\rho}(0)]+g_{p \alpha}^{*} \mathrm{~L}_{\mathbf{F}_{\alpha}, \epsilon \mathbf{G}_{p}}[\tilde{\rho}(0)]\right)\right]\right] .
\end{aligned}
$$

Now, the DF condition, Eq. (4.1), implies that the DF error generators commute with the DF density matrix: $\left[\mathbf{F}_{\alpha}, \tilde{\rho}(0)\right]=0$. We also again assume perfect quantum memory [Eq. 4.13 )]. Thus, for an arbitrary operator $\mathbf{A} \in \mathcal{A}(\mathcal{H})$, we find that we can commute the initial density matrix through the operator $\mathrm{L}$ :

$$
\begin{aligned}
\mathrm{L}[\tilde{\rho}(0) \mathbf{A}] & =-\frac{i}{\hbar}[\mathbf{H}, \tilde{\rho}(0) \mathbf{A}]+\frac{1}{2} \sum_{\alpha, \beta=1}^{M} a_{\alpha \beta}\left(2 \mathbf{F}_{\alpha} \tilde{\rho}(0) \mathbf{A} \mathbf{F}_{\beta}^{\dagger}-\mathbf{F}_{\beta}^{\dagger} \mathbf{F}_{\alpha} \tilde{\rho}(0) \mathbf{A}-\tilde{\rho}(0) \mathbf{A} \mathbf{F}_{\beta}^{\dagger} \mathbf{F}_{\alpha}\right) \\
& =-\frac{i}{\hbar} \tilde{\rho}(0)[\mathbf{H}, \mathbf{A}]+\frac{1}{2} \sum_{\alpha, \beta=1}^{M} a_{\alpha \beta} \tilde{\rho}(0)\left(2 \mathbf{F}_{\alpha} \mathbf{A} \mathbf{F}_{\beta}^{\dagger}-\mathbf{F}_{\beta}^{\dagger} \mathbf{F}_{\alpha} \mathbf{A}-\mathbf{A} \mathbf{F}_{\beta}^{\dagger} \mathbf{F}_{\alpha}\right) \\
& =\tilde{\rho}(0) \mathrm{L}[\mathbf{A}]
\end{aligned}
$$

so that:

$$
\mathrm{L}^{(n-1)}[\tilde{\rho}(0) \mathbf{A}]=\tilde{\rho}(0) \mathrm{L}^{(n-1)}[\mathbf{A}] ; \quad \mathrm{L}^{(n-1)}[\mathbf{A} \tilde{\rho}(0)]=\mathrm{L}^{(n-1)}[\mathbf{A}] \tilde{\rho}(0)
$$

Examining the contribution to the decoherence rates from the Lamb shift term, we thus find that

$$
\left(\frac{1}{\tau_{n}^{(1)}}\right)_{\text {Lamb }}^{n}=-\frac{i}{\hbar} \operatorname{Tr}\left[\tilde{\rho}(0)\left[\mathrm{L}^{(n-1)}\left[\epsilon \mathbf{H}_{\text {Lamb }}\right], \tilde{\rho}(0)\right]\right]=0
$$

which vanishes by cycling under the trace. Next, the contribution to the decoherence rates due to the symmetry breaking perturbing error generators in Eq. (4.19) are given by 


$$
\left(\frac{1}{\tau_{n}^{(1)}}\right)_{\mathrm{SBP}}^{n}=\frac{1}{2} \sum_{p=1}^{P} \sum_{\alpha=1}^{M} \operatorname{Tr}\left\{\tilde{\rho}(0) \mathrm{L}^{(n-1)}\left[g_{p \alpha} \mathrm{L}_{\epsilon \mathbf{G}_{p}, \mathbf{F}_{\alpha}}[\tilde{\rho}(0)]+g_{p \alpha}^{*} \mathrm{~L}_{\mathbf{F}_{\alpha}, \epsilon \mathbf{G}_{p}}[\tilde{\rho}(0)]\right]\right\} .
$$

Expanding the first of these terms,

$$
\begin{aligned}
& \frac{\epsilon}{2} \sum_{p=1}^{P} \sum_{\alpha=1}^{M} g_{p \alpha} \operatorname{Tr}\left[\tilde{\rho}(0) \mathrm{L}^{(n-1)}\left[2 \mathbf{G}_{p} \tilde{\rho}(0) \mathbf{F}_{\alpha}^{\dagger}-\mathbf{F}_{\alpha}^{\dagger} \mathbf{G}_{p} \tilde{\rho}(0)-\tilde{\rho}(0) \mathbf{F}_{\alpha}^{\dagger} \mathbf{G}_{p}\right]\right] \\
= & \frac{\epsilon}{2} \sum_{p=1}^{P} \sum_{\alpha=1}^{M} g_{p \alpha} \operatorname{Tr}\left[\tilde{\rho}(0)\left(\mathrm{L}^{(n-1)}\left[2 \mathbf{G}_{p} \mathbf{F}_{\alpha}^{\dagger}\right] \tilde{\rho}(0)-\mathrm{L}^{(n-1)}\left[\mathbf{F}_{\alpha}^{\dagger} \mathbf{G}_{p}\right] \tilde{\rho}(0)-\tilde{\rho}(0) \mathrm{L}^{(n-1)}\left[\mathbf{F}_{\alpha}^{\dagger} \mathbf{G}_{p}\right]\right)\right] \\
= & \epsilon \sum_{p=1}^{P} \sum_{\alpha=1}^{M} g_{p \alpha} \operatorname{Tr}\left[\tilde{\rho}(0) \mathrm{L}^{(n-1)}\left[\left[\mathbf{G}_{p}, \mathbf{F}_{\alpha}^{\dagger}\right]\right] \tilde{\rho}(0)\right] \\
= & \epsilon \sum_{p=1}^{P} \sum_{\alpha=1}^{M} g_{p \alpha} \operatorname{Tr}\left[\mathrm{L}^{(n-1)}\left[\tilde{\rho}(0)\left[\mathbf{G}_{p}, \mathbf{F}_{\alpha}^{\dagger}\right] \tilde{\rho}(0)\right]\right]
\end{aligned}
$$

Using $\mathbf{F}_{\alpha}^{\dagger} \tilde{\rho}(0)=c_{\alpha}^{*} \tilde{\rho}(0)$, we see that $\tilde{\rho}(0)\left[\mathbf{G}_{p}, \mathbf{F}_{\alpha}^{\dagger}\right] \tilde{\rho}(0)=\tilde{\rho}(0)\left(\mathbf{G}_{p} c_{\alpha}^{*}-c_{\alpha}^{*} \mathbf{G}_{p}\right) \tilde{\rho}(0)$ and thus this term vanish. Similar reasoning implies the vanishing of the second term in Eq. (4.22). Thus we have proven that $1 / \tau_{n}^{(1)}=0$ : under Markovian evolution, DF subspaces are completely stable to first order under a symmetry breaking perturbation [36].

\section{THE DYNAMICAL FIDELITY}

The results derived in the previous Section imply that DF subspaces are robust to small perturbations when the DF subspace is operating as a quantum memory. In order to address what happens when perturbations are made on the system as it evolves according to some desired quantum computation, we have to first define an analog of the mixed-state memory fidelity for an evolving system. This is

$$
F_{\mathrm{d}}(t)=\operatorname{Tr}\left[\rho_{U}(t) \rho(t)\right],
$$

where $\rho_{U}(t)$ is the desired unitary evolution

$$
\rho_{U}(t)=\mathbf{U}_{S}(t) \rho(0) \mathbf{U}_{S}^{\dagger}(t), \quad \text { with } \quad \mathbf{U}_{S}(t)=\exp \left[-\frac{i}{\hbar} \mathbf{H}_{S} t\right] .
$$

Here $\mathbf{H}_{S}$ is the system Hamiltonian, and may include a "program" Hamiltonian which implements a quantum algorithm on the system. This dynamical fidelity is a good measure of the difference between the desired evolution of the system and the actual, "noisy" evolution. Thus, $0 \leq F_{\mathrm{d}}(t) \leq 1$, with $F_{\mathrm{d}}(t)=1$ if and only if the evolution is perfect, i.e., $\rho(t)=\rho_{U}(t)$.

The decoherence rates for the dynamical fidelity are defined in the same manner as for the memory fidelity:

$$
F_{\mathrm{d}}(t)=\sum_{n} \frac{1}{n !}\left(\frac{t}{\bar{\tau}_{n}}\right)^{n}: \frac{1}{\bar{\tau}_{n}}=\left\{\operatorname{Tr}\left[\left\{\rho_{U}(t) \rho(t)\right\}^{(n)}\right]\right\}^{1 / n} .
$$

\section{A. Markovian Case}

First we consider the dynamical fidelity within the context of the Markovian limit, using the SME approach. We restrict our attention as before to DF subspaces, so the density matrix $\tilde{\rho}$ satisfies Eq. (2.15) with $\mathrm{L}_{D}[\tilde{\rho}(t)]=0$. We then imagine this DF subspace to be perturbed by a symmetry-breaking perturbation: $\mathrm{L}_{D} \mapsto \mathrm{L}_{D}^{\prime}$, where the perturbed density matrix satisfies the following SME:

$$
\frac{\partial \tilde{\rho}}{\partial t}=-\frac{i}{\hbar}\left[\mathbf{H}_{S}, \tilde{\rho}(t)\right]+\mathrm{L}_{D}^{\prime}[\tilde{\rho}(t)]
$$


Similarly to Eq. (4.8) (see also Ref. [10]), the new terms in this SME are given by:

$$
\mathrm{L}_{D}^{\prime}[\tilde{\rho}(t)]=\sum_{\alpha=1}^{M} \sum_{p=1}^{P}\left(a_{\alpha p} \mathrm{~L}_{\mathbf{F}_{\alpha}, \epsilon \mathbf{G}_{p}}[\tilde{\rho}(t)]+a_{\alpha p}^{*} \mathrm{~L}_{\epsilon} \mathbf{G}_{p}, \mathbf{F}_{\alpha}[\tilde{\rho}(t)]\right)+O\left(\epsilon^{2}\right) .
$$

The perturbed first order dynamical fidelity decoherence rate is given by:

$$
\frac{1}{\bar{\tau}_{1}}=\operatorname{Tr}\left[\tilde{\rho}_{U}(0) \frac{\partial \tilde{\rho}(0)}{\partial t}+\frac{\partial \tilde{\rho}_{U}(0)}{\partial t} \tilde{\rho}(0)\right] .
$$

The first of these terms vanishes via the arguments given for the memory fidelity (essentially, since $\mathbf{F}_{\alpha} \tilde{\rho}=c_{\alpha} \tilde{\rho}$ by the DF subspace property). The second term also vanishes, by permutation of the trace after using $\partial \rho_{U}(0) / \partial t=$ $-i \hbar\left[\mathbf{H}_{S}, \rho(0)\right]$. Thus we find that DF subspaces are stable to first order in time also when the system is allowed to evolve.

Further, it is easy to see that the higher order dynamic fidelities now do not vanish. For example, the second order dynamic decoherence rate contains terms like $\left[\mathbf{H}_{S}, \rho(0)\right] \mathrm{L}_{D}^{\prime}[\rho(t)]_{t=0}$, which do not allow the simple permutation of the trace.

\section{B. Non-Markovian Case}

Is there an analogous result for the non-Markovian situation? We can address this within the OSR. The arguments of Sec. III A showed that the first order decoherence rate will always vanish, and so according to the arguments given above, this applies also to the dynamical fidelity. Examining the second order decoherence rates, we find:

$$
\left(\frac{1}{\bar{\tau}_{2}}\right)^{2}=\operatorname{Tr}\left[\hbar^{2}\left[\mathbf{H}_{S},\left[\mathbf{H}_{S}, \tilde{\rho}(0)\right]\right] \tilde{\rho}(0)+\left[\mathbf{H}_{S}, \tilde{\rho}(0)\right] \mathrm{L}^{\prime}(t)[\tilde{\rho}(0)]_{t=0}+\tilde{\rho}(0)\left\{\frac{\partial \mathrm{L}^{\prime}(t)}{\partial t}[\tilde{\rho}(0)]\right\}_{t=0}\right],
$$

where $\mathrm{L}^{\prime}$ is now given by Eq. (4.8). As in the semigroup analysis above, the second of these traces does not vanish (nor does the first, but it will be cancelled due to a contribution from the third).

Thus we find that for the dynamical fidelity, the effect of a symmetry breaking perturbation results in a second order time instability in the system for both Markovian and non-Markovian situations. It is remarkable that while the memory fidelity is completely robust to first order $(\epsilon)$ perturbing errors, the dynamical fidelity does not show the same robustness, with instability arising at second order in time. In terms of real quantum computation therefore, this implies that DF subspaces must be supplemented by QECC in order to be truly useful beyond merely providing high quality quantum memory. In particular, the instability in the dynamical fidelity implies that in order to realize the robustness of DF subspaces to symmetry breaking perturbations, operations on the DF subspace must be performed over a time scale short in comparison with the perturbing error rate. Thus, for the realistic scheme in which DF subspaces are supplemented by quantum error correcting codes (by, for example, concatenating the DF subspaces within QECC as in Ref. [11]), if the operations performed on the DF subspace in order to execute the QECC are executed frequently and rapidly, the full scheme can provide a significant improvement over pure DF subspaces.

\section{SUMMARY AND CONCLUSIONS}

We have shown here how the formally exact operator sum representation (OSR) for the time evolution of the density matrix can be cast in a form which bears a significant resemblance to the semigroup master equation (SME), through the introduction of a time-independent (fixed) operator basis. By using this fixed-basis OSR equation, we were able to easily calculate the fidelity in the OSR, as well as to provide a derivation of the SME which makes explicit the role played by the coarse-graining assumption. Somewhat surprisingly, we found an important difference between the OSR and the SME, namely, the first order decoherence rate always vanishes in the former, but not always in the latter, for a finite Hamiltonian. This effect is readily traced to the coarse-graining time averaging assumption within our derivation. This result is significant for both error-correction schemes aimed at improving the fidelity, and for commonly used simplified models of decoherence. We illustrated the latter with the well-known case of phase-damping.

Using the fixed-basis OSR, we have then undertaken a detailed study of short-time expansions of the mixedstate fidelity under a variety of conditions. The mixed-state fidelity provides a measure of the extent of quantum coherence in the system. We have examined both the usual mixed-state memory fidelity relevant to quantum memory, 
and a dynamical fidelity which we defined to act as a measure of coherence for a time-evolving system. Our main achievement here has been to extend the robustness results of Ref. 10] regarding decoherence-free (DF) subspaces. For the preservation of quantum memory, we showed that in both the OSR and SME approaches, DF subspaces are stable to all orders in time to a symmetry-breaking perturbation. The first errors entering the quantum memory can therefore only be $O\left(\epsilon^{2}\right)$, where $\epsilon$ measures the strength of the perturbation. This result goes beyond the first order stability result of $O\left(\epsilon t^{2}\right)$ arrived at in Ref. [10], which was restricted to the Markovian case. It shows that $D F$ subspaces are indeed ideal for quantum memory in all situations. In making this statement, we note that we have not shown here how to perform input and output to the DF subspaces. Further investigation is needed to address this issue. The quantum memory stability results are summarized in Table If. For the dynamical fidelity, a weaker result is obtained, namely that this has only a vanishing first order decoherence rate under a perturbation. Thus the first errors entering the dynamical fidelity can be $O\left(\epsilon t^{2}\right)$.

This stability analysis of static and dynamic fidelities is of particular relevance for practical implementations of quantum computation. The complete stability to perturbations of static fidelity within DF subspaces is very encouraging for use in quantum memory. Thus passive error correction appears to be sufficient for this. In contrast, the weaker first order stability condition derived for the dynamical fidelity within DF subspaces implies that application of active error-correction techniques will likely be necessary in order to preserve coherence during computation. Further, if the operations performed on the DF subspace in order to execute active error-correction are executed rapidly, then the full DF stability to symmetry breaking perturbations can be recovered. Such a scheme of combining active and passive error-correction is possible by concatenating codes constructed from DF subspaces with active quantum error correction, as demonstrated in Ref. 11.

\section{ACKNOWLEDGMENTS}

This material is based upon work supported by the U.S. Army Research Office under contract/grant number DAAG55-98-1-0371. We would like to thank Dr. I. Chuang for helpful discussions and Dr. P. Zanardi for useful comments. Part of this work was completed during the Elsag-Bailey - I.S.I. Foundation research meeting on quantum computation in 1998.

TABLE I. Different order decoherence rates for the mixed-state memory $(\tau)$ and dynamical $(\bar{\tau})$ fidelities under the various conditions cited in the text. Unless otherwise noted, $n \geq 1$.

\begin{tabular}{lcc}
\hline \hline & SME & OSR \\
\hline General & $1 / \tau_{1} \neq 0$ & $1 / \tau_{1}=0^{\mathrm{a}}$ \\
& $1 / \tau_{n} \neq 0, n \geq 2$ & $1 / \tau_{n} \neq 0, n \geq 2$ \\
DF subspaces & $1 / \tau_{n}=0^{\mathrm{b}}$ & $1 / \tau_{n}=0$ \\
memory fidelity for $\epsilon$-perturbed DF subspaces & $1 / \tau_{n}=0$ & $1 / \tau_{n}=0$ \\
dynamical fidelity for $\epsilon$-perturbed DF subspaces & $1 / \bar{\tau}_{1}=0$ & $1 / \bar{\tau}_{1}=0$ \\
& $1 / \bar{\tau}_{n} \neq 0, n \geq 2$ & $1 / \bar{\tau}_{n} \neq 0, n \geq 2$ \\
\hline \hline
\end{tabular}

afinite bath and finite total Hamiltonian only.

both with and without perturbing Lamb shift. 
[1] A.M. Steane, Rep. on Prog. in Phys. 61, 117 (1998), LANL preprint quant-ph/9708022.

[2] W.G. Unruh, Phys. Rev. A 51, 992 (1995).

[3] S. Haroche and J.M. Raimond, Physics Today 49, 51 (1996).

[4] R. Landauer, Phys. Lett. A 217, 188 (1996).

[5] C.H. Bennett and P.W. Shor, IEEE Trans. Info. Theory 44, 2724 (1998).

[6] L.-M Duan and G.-C. Guo, Phys. Rev. A 57, 737 (1998).

[7] P. Zanardi and M. Rasetti, Mod. Phys. Lett. B 11, 1085 (1997), LANL preprint quant-ph/9710041.

[8] P. Zanardi and M. Rasetti, Phys. Rev. Lett. 79, 3306 (1997), LANL preprint quant-ph/9705044.

[9] P. Zanardi, Phys. Rev. A 57, 3276 (1998), LANL preprint quant-ph/9705045.

[10] D.A. Lidar, I.L. Chuang and K.B. Whaley, Phys. Rev. Lett. 81, 2594 (1998), LANL preprint quant-ph/9807004.

[11] D.A. Lidar, D.A. Bacon and K.B. Whaley, Concatenating Decoherence Free Subspaces with Quantum Error Correcting Codes, LANL preprint quant-ph/9809081.

[12] K. Kraus, States, Effects and Operations, Fundamental Notions of Quantum Theory (Academic, Berlin, 1983).

[13] A.J. van Wonderen and K. Lendi, J. Stat. Phys. 80, 273 (1995).

[14] G. Lindblad, Commun. Math. Phys. 48, 119 (1976).

[15] R. W. Zwanzig, Statistical Mechanics of Irreversibility, Vol. 3 of Lectures in Theoretical Physics (Interscience, New York, 1961), p. 106.

[16] R. L. E. Knill and W. Zurek, Science 279, 342 (1998).

[17] D. Aharonov and M. Ben-Or, in Proceedings of 29th Annual ACM Symposium on Theory of Computing (STOC) (ACM, New York, NY, 1997), p. 46, LANL preprint quant-ph/9611025.

[18] P. Zanardi, Computation on a noiseless quantum code and symmetrization, LANL preprint quant-ph/9901047.

[19] B. Schumacher, Phys. Rev. A 54, 2614 (1996).

[20] R. Alicki and K. Lendi, Quantum Dynamical Semigroups and Applications, No. 286 in Lecture Notes in Physics (SpringerVerlag, Berlin, 1987).

[21] I.L. Chuang and M.A. Nielsen, J. Mod. Optics 44, 2455 (1997).

[22] P. Pechukas, Phys. Rev. Lett. 73, 1060 (1994).

[23] E.B. Davies, Commun. Math. Phys. 39, 91 (1974).

[24] E.B. Davies, Math. Ann. 219, 147 (1976).

[25] P. Beck and K. Lendi, Phys. Rev. A 47, 346 (1993).

[26] R. Jozsa, J. Mod. Optics 41, 2315 (1994).

[27] C.H. Bennett, D.P. DiVincenzo, J.A. Smolin and W.K. Wootters, Phys. Rev. A 54, 3824 (1996).

[28] L.-M. Duan and G.-C. Guo, Phys. Rev. A 56, 4466 (1997), LANL preprint quant-ph/9706020.

[29] D. Vitali and P. Tombesi, LANL preprint quant-ph/9802033.

[30] P. Zanardi and F. Rossi, LANL preprint quant-ph/9804016.

[31] The vanishing of the first order decoherence time is well known in the exact Hamiltonian framework: J. I. Kim, M. C. Nemes, A. F. D. de Toledo Piza and H. E. Borges, Phys. Rev. Lett. 77, 207 (1996). However, our calculation of this effect in the OSR seems to be new, as well as the observation that this is related to the finiteness of the system part of the system-bath interaction.

[32] I.L. Chuang and Y. Yamamoto, Phys. Rev. A 55, 114 (1997), LANL preprint quant-ph/9704030.

[33] E. Knill and R. Laflamme, Phys. Rev. A 55, 900 (1997).

[34] R. Silbey and R.A. Harris, J. Phys. Chem. 93, 7062 (1989).

[35] R. Kosloff, M.A. Ratner and W.B. Davis, J. Chem. Phys. 106, 7036 (1997).

[36] An interesting example of this general first-order stability is provided by the recently analysed situation of environmentally induced superselection of energy eigenstates for a Brownian particle interacting with an adiabatically slow bath of harmonic oscillators [37.

[37] J.P. Paz, W.H. Zurek, LANL preprint quant-ph/9811026. 